\title{
A Topic-based Computer Aided Instruction System for Programming Practice Courses
}

\author{
Yan ZHANG, Xiaoli ZHANG, Kejun ZHANG, Deli SHEN \\ Department of Computer Science and Technology, Beijing Electronic Science and Technology Institute, \\ Beijing, P. R. China
}

\begin{abstract}
For overcoming the lack of systematicness and comprehensiveness of teaching contents in the micro-lecture, a topic-based computer aided instruction (CAI) system is designed and implemented. The CAI system is a web application based on Browser/Server framework. A topic amounts to a micro-lecture. By organizing and presenting teaching contents from diverse perspectives, the CAI system can make educatees to learn the inherent relation among the knowledge points and construct a framework of knowledge in educatees' brain. The CAI system can be used in flipped classroom to improve the quality and the effect of teaching.

KEYWORD: Flipped classroom; Micro-lecture; Computer aided instruction
\end{abstract}

\section{INTRODUCTION}

The flipped classroom [1], which is a very interesting education mode nowadays, helps improving the sense of problematic and innovation for students, and fostering the habit and the ability of self-exploration for them. The micro-lecture [2] is an important element for the flipped classroom. By using some elaborate micro-lectures, teachers can improve the quality and the effect of teaching in a flipped classroom. The micro-lecture has significant characteristics. One is shorter teaching time, generally five to eight minutes. The other is less content of courses, generally focusing on one knowledge. Therefore, the micro-lecture does not own complex curricula architectures and numerous teaching goals. The fragmentation of teaching in micro-lecture is very appropriate for self-learning in spare time. However, the teaching of micro-lectures lacks the systematicness and comprehensiveness.

A college education should not only develop the ability of solving some specific problem for educatees, but also establish a systematic framework of some domain knowledge for them. The microlecture is not competent for this task. How do we remedy the demerit of micro-lectures? By Web technology, we design and implement a topic-based computer aided instruction (CAI) system for programming practice courses (PPC) [3], named BESTI PPC Platform. A topic amounts to a knowledge point, and a knowledge point corresponds to a micro-lecture. In BESTI PPC Platform, users can learn every topic, and all related topics can be reorganized in different pattern for users. Thus, BESTI PPC Platform realizes that a teaching content can be presented in different perspectives in terms of different patterns. With BESTI PPC Platform, students can learn inherent relations among knowledge points, differences between confusable knowledge points and the use of every knowledge point as well as the knowledge points themselves. As a result, BESTI PPC Platform establishes a framework of knowledge for students who use it to learn PPC.

The remainder of this paper is organized as follows. Section 2 describes the design of BESTI PPC Platform from its function, architecture and database. Section 3 presents the implementation of the platform and used techniques. Finally, we conclude this paper in Section 4.

\section{DESIGN OF BESTI PPC PLATFORM}

BESTI PPC Platform is a Web application based on Browser/Server $(\mathrm{B} / \mathrm{S})$ framework. Teachers upload and maintain the learning materials, and students log in the platform and study these materials on their own.

\subsection{Function design}

The function of the platform can be divided to three parts. 


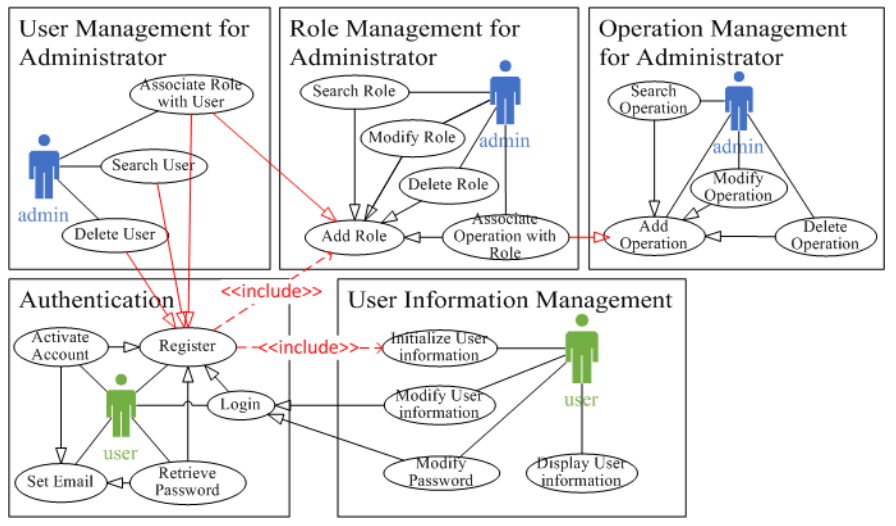

(a) Management of actors

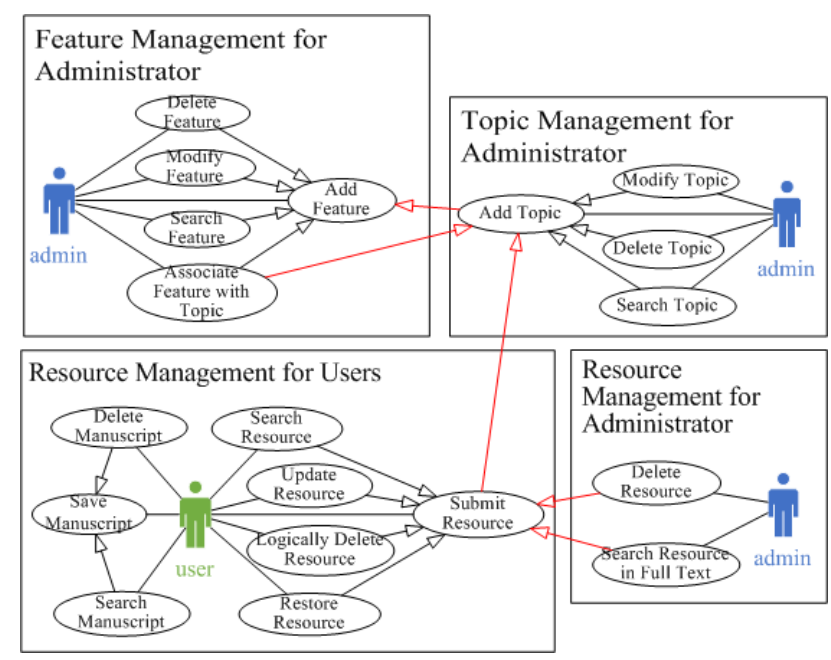

(b) Management of content

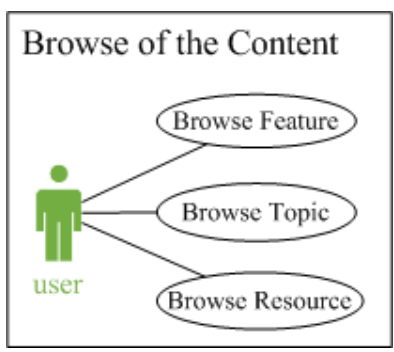

(c) Browse of Content

Figure 1. Use case diagrams of BESTI PPC Platform.

The first one is the management of actors, which is responsible for user identity authentication, access control and maintenance of personal information. Figure 1a gives the use case diagram of this part.

The second part, the crucial component of the platform, is the management of content, whose use case diagram is shown in Figure 1b. For associating all relevant micro-lectures and organizing learning materials from diverse perspectives, we extract three properties for all learning materials, that is, topic, resource and feature. The topic corresponds to the knowledge point, e.g. function in a programming language. When the content of a topic is very big, it can be divided into many sub-topic. For example, the topic of function may include some sub-topics such as function definition, function invocation and parameter passing. The resource is the element of the topic, and it is a refinement of the teaching content in a topic. Commonly, reference, as a kind of parameter passing mode, is taught from the syntax of reference, the semantic of reference, the instance of reference, the difference between reference and value passing, and so on, every one of which is a resource. The feature identifies an attribute of a topic or resource. Generally, there are features such as syntax, semantic, concept, code, figure and table. A feature may include some sub-feature. For example, syntax may have definition (or BNF), description and instance sub-features. The third part is the browse of content, whose use case diagram is shown in Figure 1c.

According to the analysis of use case, we elicit the functional modules of BESTI PPC Platform as followings:

- Basic service. It checks user identity and maintains the dictionary data used in this platform.

- User operations. It consists of resource display, which presents for users the teaching contents in the platform, and content management, which provide for teachers the upload, edition and maintenance of teaching contents.

- Administrator operation. It maintains the topics, resources, features, users and roles respectively.

- Search. It performs full text retrieval in exact or fuzzy match mode or

- Others. It includes new update, hot topics, news, related website, and so on, some of which is referred from existed systems.

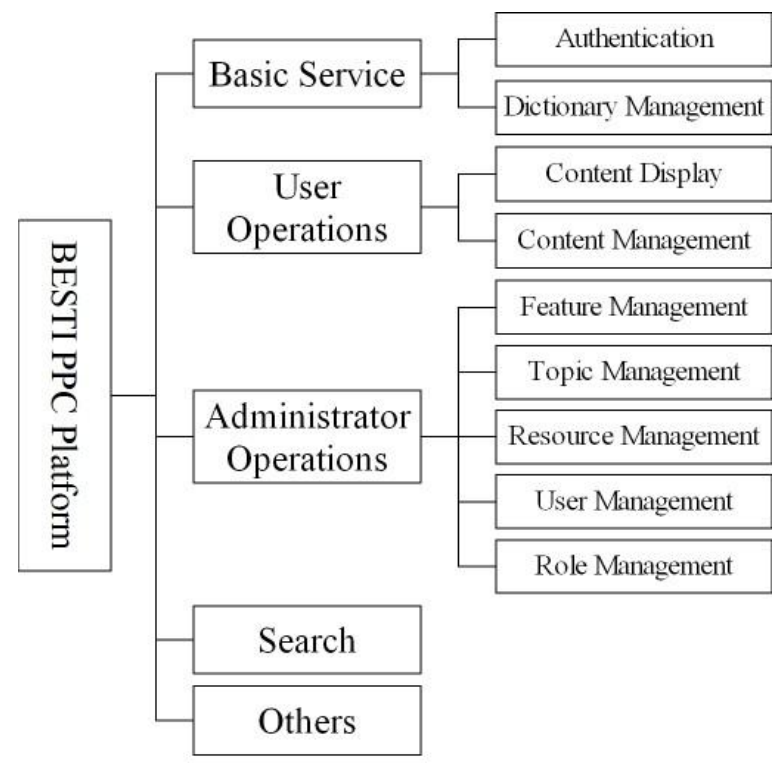

Figure 2. Function modules of BESTI PPC Platform.

\subsection{Architecture design}

Model View Controller (MVC) [4] is a paradigm for software design, which builds up a system by separating the business logic, data and display of interface. Since MVC can meet low coupling and high reusability of system, we adopt MVC to develop BESTI PPC Platform. 
The architecture of BESTI PPC Platform is shown in Figure 3. The presentation layer is responsible for statically or dynamically displaying teaching contents and fetching input data from users. The Controller layer transmits the command and data input by users to the service layer. This layer also control which information should be returned to users. The service layer takes the main tasks. According to the separation of concerns, the service layer includes six modules, which are responsible for validating the inputs from above layer, validating the user's operations based on his role, dealing with transactions, protecting the data in the system against misuse, management of multi-user concurrent access and organization of contents respectively. The data connection layer performs the connection between the service layer and the database that store the teaching contents and users information.

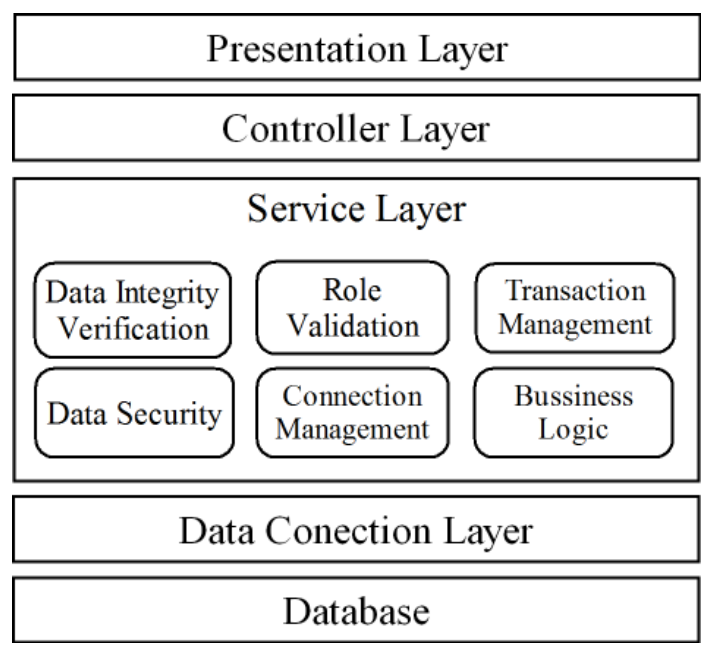

Figure 3. Architecture of BESTI PPC Platform

\subsection{Database design}

Database is the crucial component of the platform. The entity-relation (ER) diagram of the database is shown in Figure 4. The database design follows two principles. One is to reduce the coupling between entities. The other is to cut down redundant entities. For the first principle, we create explicit association between entities if they have some relation to enhance the dependability of entities. For the second principle, we use a foreign key instead of a relation table when there is a one to one (or one to many) relation. There are thirteen entity tables and five relation tables. The entity table is divided two categories, i.e. entity tables for business and entity tables for records.

Based on Role-Based Access Control (RBAC), the authentication is designed in the database. There are three roles in the system, i.e., administrator, teacher and student. The administrator manages the system. The teacher maintains teaching contents in the system. The student browses over the teaching contents.

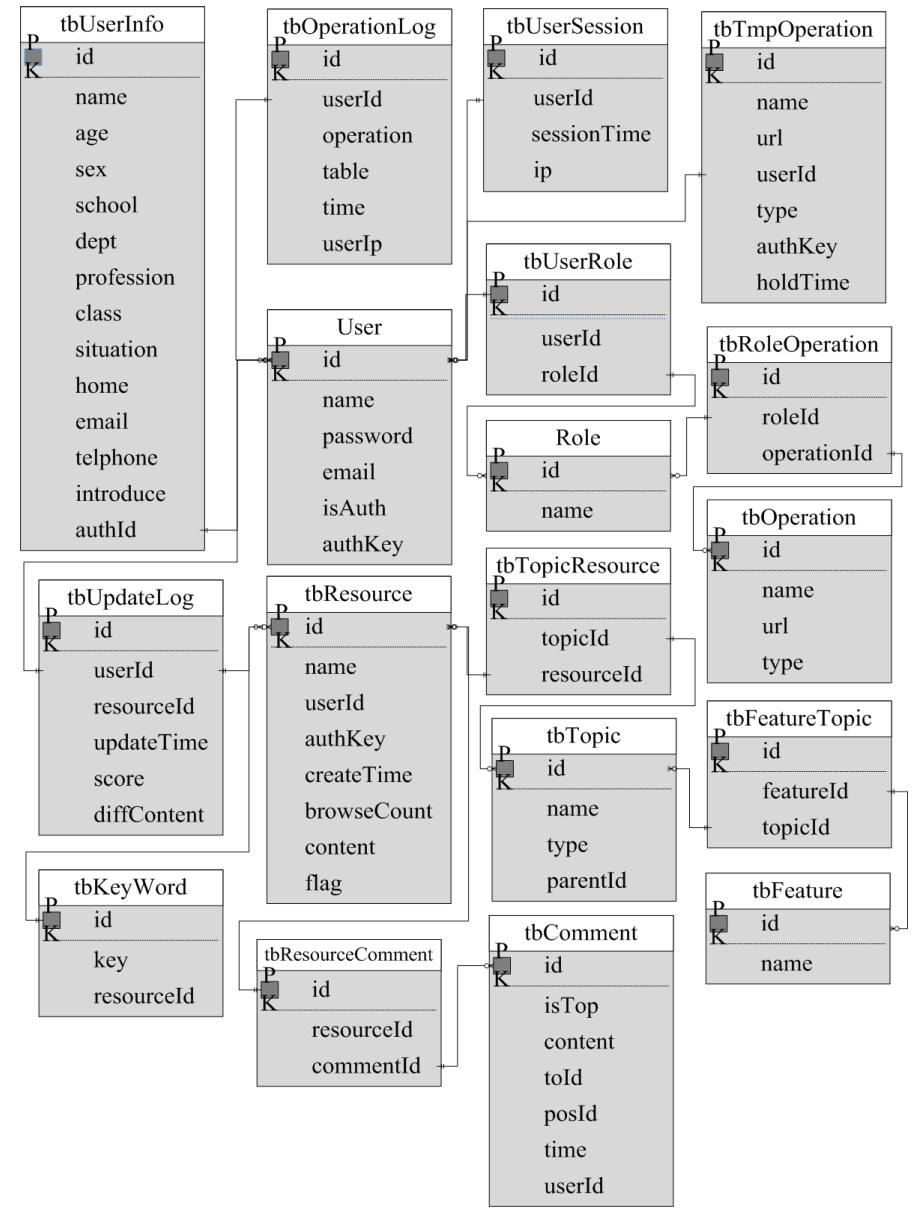

Figure 4. ER Diagram of the database in BESTI PPC Platform

\section{IMPLEMENTATION OF BESTI PPC PLATFORM}

We had implemented BESTI PPC Platform with JDK1.7 in Eclipse integrated development environment, where Maven was used to manage packages. Particularly, controllers had been implemented with SpringMVC; business logic had been realized with Spring [5] and data connection layer had been materialized with Mybatis [6]. Figure 5 gives the main page of BESTI PPC Platform.

\subsection{Database and data connection layer implementation}

MySQL is an open source relational database management system. Because of the small size and high performance of MySOL, we had used it to implement the database in BESTI PPC Platform.

In the data connection layer, we had implemented create, retrieve, update and delete operations for database with Mybatis. Mybatis is a Java persistence framework, which simplifies coding compared to JDBC because Mybatis maps Java methods to SQL statements. Unlike Hibernate, Mybatis does not depend on the third-party products and rarely use reflection mechanism, which can provide better performance. Mybatis also provides declarative data 
caching and supports dynamic SQL statements. Therefore, we adopted Mybatis.

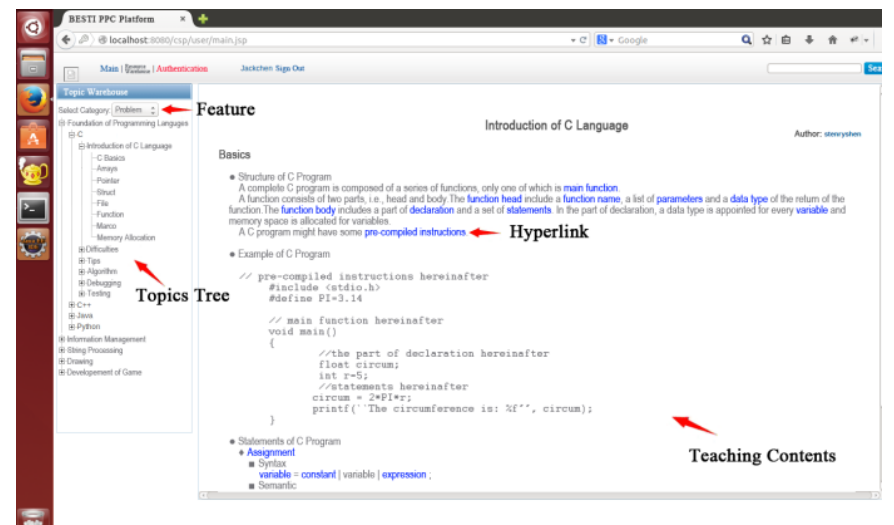

Figure 5. Main page of BESTI PPC Platform

\subsection{Service layer implementations}

Based on Representational State Transfer (REST) and interface-oriented programming, we had implemented a set of highly extensible interfaces in the service layer, which are listed in Table 1. All input or output parameters of these interfaces are objects instantiated from predefined model classes.

Table 1. Interfaces in the service layer.

\begin{tabular}{|l|l|}
\hline Name & Description \\
\hline AuthServiceI & Interface for authentication \\
\hline FeatureServiceI & Interface for feature service \\
\hline InfoServiceI & Interface for information service \\
\hline KeyWordServiceI & Interface for keyword service \\
\hline OperationServiceI & Interface for operation service \\
\hline RoleServiceI & Interface for role service \\
\hline ResourceServiceI & Interface for resource service \\
\hline TopicServiceI & Interface for topic service \\
\hline UserSessionServiceI & Interface for user session \\
\hline UserServiceI & Interface for user service \\
\hline ViewServiceI & Interface for display of content \\
\hline
\end{tabular}

\subsection{Presentation layer implementation}

The webpages in BESTI PPC Platform had been implemented based on DIV +CSS standard, which separate the content of webpages with their presentation. We used Jquery [7] to realize the interaction and dynamic request of webpages, since Jquery support dynamic request, partial page update, and asynchronous load.

\section{CONCLUSION}

For overcoming the disadvantages of the microlectures, we design and implement a topic- based CAI system for programming practice courses based on web technology. In the system, the topics, which focuses on explaining a knowledge point, equals to a micro-lecture. By refining the teaching content of a micro-lecture and extracting attributes from all teaching contents, we realize that the teaching content in the system can be organized and presented from diverse perspectives. As a result, the system can teach not only individual knowledge points, but also the inherent relation among the knowledge points, and can construct a framework of knowledge in students' brain. By enhancing the systematicness and comprehensiveness of the micro-lecture, our system can be used for the flipped classrooms.

\section{REFERENCES}

[1] Lage, M., Platt, G. \& Treglia, M. 2000. Inverting the classroom: a gateway to creating an inclusive learning environment. Journal of Economic Education 31(1): 3043.

[2] McGrew, L.A. 1993. A 60-second course in organic chemistry. Journal of Chemistry Education 70 (7): 543544.

[3] Zhang, X., Zhou, Z., Zhang, Y. \& Zhao G. 2012. Programming Practice Tutorial. Beijing: Tsinghua University Press.

[4] Reenskaug, T. 1979. THING-MODEL-VIEW-EDITOR: an example from a planning system. Xerox PARC Technical Note.

[5] Johnson, R. 2002 Expert One-on-one J2EE development and design. Birmingham: Wrox.

[6] Mudunuri, S. 2013. Mybatis in practice: a step by step approach for learning Mybatis framework. Luxembourg: Createspace.

[7] Duckett, J. 2014. JavaScript \& jQuery: interactive frontend web development hardcover. $\mathrm{NJ}$ : Wiley. 